\title{
Measurement of Argon emission spectral of ICP plasma using a diagnostic system based on photomultiplier tubes array
}

\author{
Weifeng Liu ${ }^{1,2}$, Yiyong Yang ${ }^{1,2,}$, Chuankun $\mathrm{Han}^{1,2}$, Jia Cheng ${ }^{3}$ and Linhong $\mathrm{Ji}^{3}$ \\ ${ }^{1}$ School of Engineering and Technology, China University of Geosciences (Beijing), Beijing 10083, China \\ ${ }^{2}$ Key Laboratory of Deep Geodrilling Technology, Ministry of Land and Resources, Beijing 100083, China \\ ${ }^{3}$ Department of Mechanical Engineering, Tsinghua University, Beijing 100084, China
}

\begin{abstract}
Optical emission spectroscopy (OES) is one of the most important diagnostic tools in plasma physics. A self-built spectroscopic diagnostic system, owning temporal and spatial resolution, has been constructed using photo multiplier tubes (PMTs) array, spectrometer and other parts. The problem of superposition between inlet plane of bundle fiber and the focal plane of the spectrometer is analyzed and solved. In addition, the synchronization regulation of output of PMTs has been completed. This system is installed on an inductively coupled (ICP) plasma chamber in order to study the Argon (Ar) emission spectrum generated from typical radio frequency (RF) and pulse discharges. The test results show that the intensity of Ar emission spectrum increases with the power and pressure, but increase less with the flow and current ratio. Under pulse discharge condition, the intensity of spectrum does not change with the frequency, neither does the broadening of spectrum with time.
\end{abstract}

\section{Introduction}

In order to understand the plasma chemistry, various plasma process diagnostic tools such as OES and Langmuir probe have been developed to quantify the concentration of reactive species in the plasma[1]. Because it is non-intrusive, inexpensive, and can be easily incorporated into an existing plasma reactor, the OES quickly gains popularity in the microelectronics industry for monitoring the plasma processing. Optical emission spectroscopy (OES) has been proved to be a powerful technique in plasma diagnostics [4-7]. There are mainly two kinds of OES methods, one is measurement of the spectrum of certain wavelengths by PMT and slit [2-5], the other is using two-dimensional detector arrays such as charge coupled device (CCD) or intensified charge-coupled device (iCCD) camera to provide the spatial multi-channel spectrum of a range of wavelengths[6,7]. The CCDs own various advantages including their subtle detector dimension, high sensitivity, and significant signal to noise ratio. However, the readout period is often longer and the time resolution is usually several $\mathrm{KHz}$, which make the CCD or iCCD has stringent restrictions on the time response of the emission spectral measurement. In many cases, the readout time $(\sim 0.5 \mathrm{~s})$ is much longer than the typical time constant of the plasma evolution; hence, temporal variation of the intensity is hardly to be obtained directly by using the spectroscopic system with a CCD or iCCD camera.

In this paper, we analyzed the development of a novel spectroscopic measurement system based on PMTs array. We successfully constructed the measurement system to measure the spatially and temporally resolved intensity of plasma light emissions from ICP Ar RF discharge and pulse discharge.

\section{Spectroscopic system}

\subsection{Spectroscopic system}

Figure 1 shows the schematic overview of the spectroscopic measurement system based on PMTs array. Emissions from an ICP plasma chamber formed from Argon gas are collimated by convex lenses and introduced to the measurement system by the inlet optical fibers with inlet core diameter of $2 \mathrm{~mm}$. The other ends of the optical fibers are bundled in a one-dimensional vertical array fixed on a six-degree-of-freedom (6-D) adjusting mount. And the one-dimensional vertical array is coupled with the entrance slit of a Czerny-Turner spectrometer (Horiba iHR550) by the 6-D adjusting mount.

\footnotetext{
${ }^{*}$ Corresponding author: yangyy@cugb.edu.cn
} 


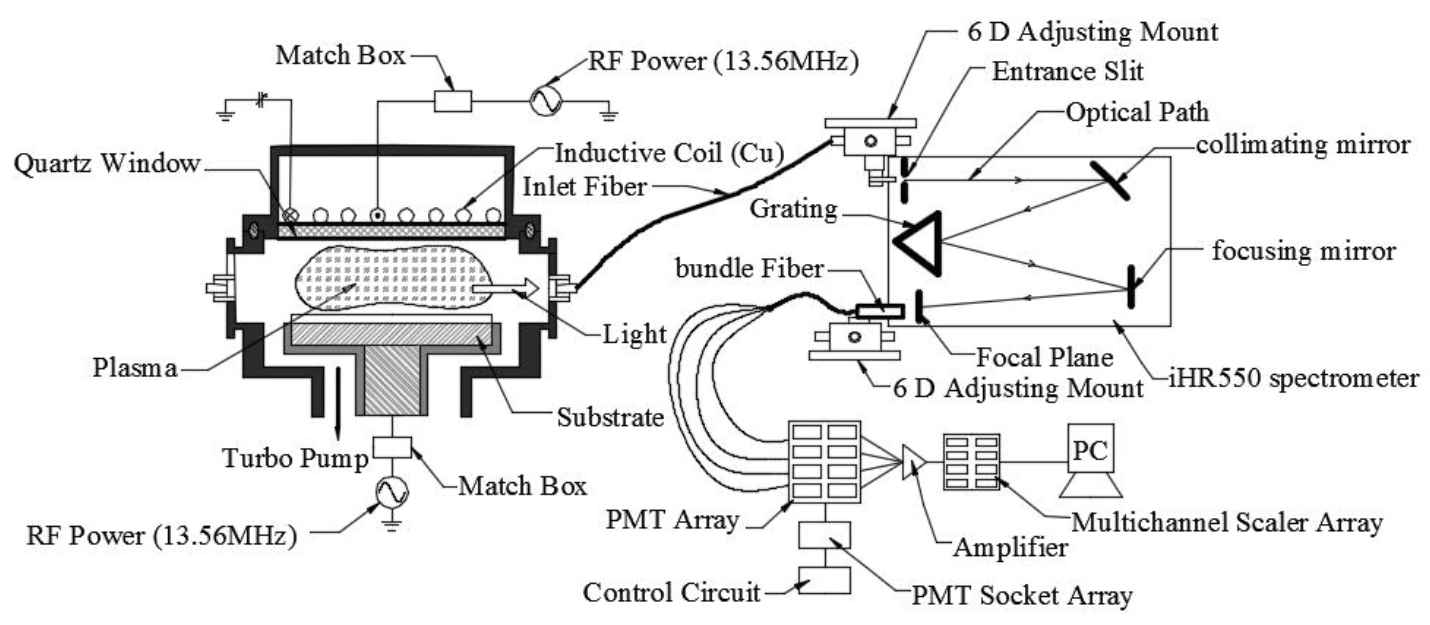

Fig.1. Schematic overview of the spectroscopic measurement system

The spectrometer has a focal length of $550 \mathrm{~mm}$ and equips three gratings of 1200,1800 and 2400 grooves $/ \mathrm{mm}$ respectively. The light from the ICP plasma chamber go into the spectrometer and then focus on the focal plane of the spectrometer outlet. Like the inlet fiber with the entrance slit, we also adjust the bundle fiber fixed on a 6-D adjusting mount to couple the inlet plane of the bundle fiber with the focal plane of the spectrometer. The inlet plane of the bundle fiber is comprised of $30 \times 30$ fibers and the outlet is comprised of 30 fibers. And each of these 30 fibers corresponds to every row of the $30 \times 30$ fibers of inlet plane. Eight of the 30 fibers are inserted into eight PMTs (HAMAMATSU R928), and each of them equips a high voltage socket (HAMAMTSU CC238). The PMTs convert the light signal from the 8 PMTs into eight channel electronic signals, and then the electronic signals are amplified by two preamplifiers (Stanford Research Systems, SR445A$350 \mathrm{MHz}$ preamplifier, 4-channel). Every channel signal amplified is collected by a multichannel scaler (MCS, ORTEC), and then analyzed by a PC software.

\subsection{Focal plane adjustment}

In order to measure the spectrum accurately, the inlet plane of the bundle fibers must be coincided with the focal plane of the spectrometer. We design a fixture to fix the inlet plane of the bundle fibers on a 6-D adjusting mount, by which the inlet plane can be moved to coincide with the focal plane. As the focal plane is still, the inlet plane is moveable by the adjusting mount. Assuming the focal plane in a fixed coordinate OXYZ and the inlet plane in a mobile coordinate $\mathrm{O}^{\prime} \mathrm{X}^{\prime} \mathrm{Y}^{\prime} \mathrm{Z}$ ' as shown in Figure 2. When the inlet plane is coincided with the focal plane, the coordinate OXYZ equals to the coordinate O'X'Y'Z'.

Except to adjust the inlet plane to coincide with the focal plane, the outlet plane of inlet fiber also should be coincided with the entrance silt of spectrometer. To obtain a higher resolution, the entrance silt should be narrow enough. Similarly, there are 6 degrees between outlet plane and entrance silt. Therefore, the vertical fiber array $1 \mathrm{~mm} \times 10 \mathrm{~mm}$ of inlet plane remains to be modulated to coincide with the entrance silt to allow most of the light into the spectrometer. We also use a 6-D adjusting mount to achieve that.

\subsection{Performance evaluation}

Accurate evaluations of total system performances, such as sensitivity, signal-to-noise $(\mathrm{S} / \mathrm{N})$ ratio, magnification are essential for appropriate usage of the spectroscopic system. Optical system setup and calibration are carried out by using sharp spectrum from laser or lamp emissions. A stable $\mathrm{Hg}$ lamb is employed to establish initial alignment of the optical components so as to minimize the unexpected noise signal. As the output of each PMT varies, the uniformity of the output in light intensity measurement must be assured. At first, we turn the grating of spectrometer to a fixed wavelength, and then we move the $\mathrm{X}$ axis of the adjustment mount to make the light reflected on the focal plane aim at the central vertical array fiber in the inlet plane of bundle fibers. After that, we insert the out fiber corresponding to the central vertical array fiber into one of the 8 PMTs in turn. Moreover, we adjust the control voltage of the control circuit and discriminator voltage of the multichannel scaler simutaneously to obtain almost same output of the eight PMTs. During the measurement process, the dark signal from surrounding evironment could enter into the system and lead to a noise signal influencing the correct signal of light. Therefore, the discriminator should be selected carefully to filter the noise from the signal. In our measurement system, due to the weak dark signal, we choose the voltage value when the signal counter is maximum as the discriminator voltage value. The last verified value of control voltage, discriminator voltage and signal counters corresponding to the eight PMTs are listed in Table 1 in details. After the initial arrangement, we make the system calibration at $\mathrm{He}-\mathrm{Ne}$ laser wavelength and then confirm that the system meets our requirement. 

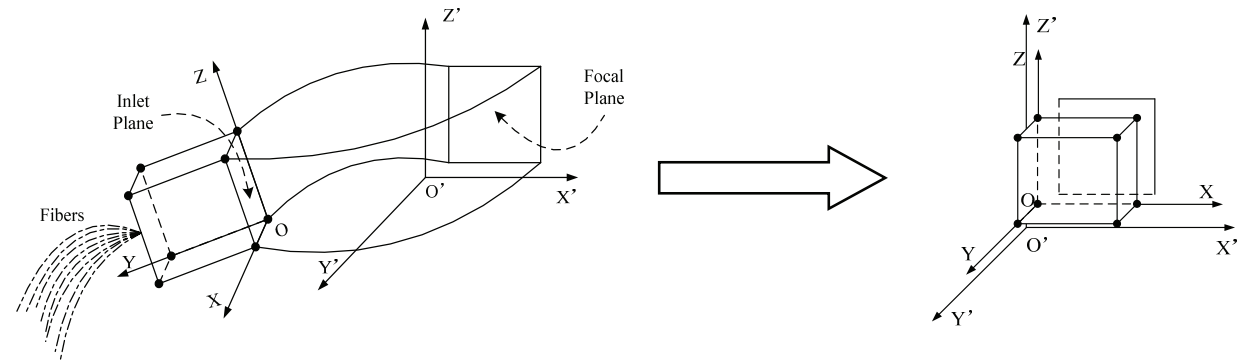

Fig.2. The coordinate of focal plane and inlet plane of bundle fibers

Table 1. Control voltage, discriminator voltage and signal counters corresponding to the eight PMTs

\begin{tabular}{|c|c|c|c|c|c|c|c|c|}
\hline PMT No. & 1 & 2 & 3 & 4 & 5 & 6 & 7 & 8 \\
\hline Disc. Vol. $^{\text {a }}$ & -0.037 & -0.04 & -0.043 & -0.049 & -0.043 & -0.049 & -0.052 & -0.052 \\
\hline Control Vol. $^{b}$ & 4.05 & 4.49 & 4.15 & 3.96 & 4.25 & 4.24 & 4.2 & 4.6 \\
\hline Counts/a.u. & 13250 & 13462 & 13617 & 13000 & 13258 & 13389 & 13520 & 13538 \\
\hline$\Delta$ Counts $^{c}$ & -129.25 & 82.75 & 237.75 & -379.25 & -121.25 & 9.75 & 140.75 & 158.75 \\
\hline$\%$ & $-0.98 \%$ & $0.61 \%$ & $1.75 \%$ & $-2.92 \%$ & $-0.91 \%$ & $0.07 \%$ & $1.04 \%$ & $1.17 \%$ \\
\hline
\end{tabular}

${ }^{a}$ Discriminator voltage

${ }^{\mathrm{b}}$ Control voltage

c $\triangle$ Counts is the difference value between each PMT's value and the average value of the eight PMTs' value.

\section{Experimental discussion}

\subsection{ICP RF discharge}

In the study of low temperature ICP argon plasma, $1 \mathrm{~s}$ and $2 p$ levels of argon atoms are often focused by researchers and processing engineers. Argon atom 1s level has higher density and more energy, which can have a great influence on active particles produced in plasma chemical reaction[8]. Due to the strong radiative transition from $2 p$ to $1 \mathrm{~s}, 2 \mathrm{p}$ level is very important for researching the argon plasma radiation properties[8]. In our ICP rf Argon discharge process, the pressure is $20 \mathrm{mT}$ Torr and the flow is $200 \mathrm{sccm}$, hence the corona model is valid for analyzing the process. The reason is that other collisional processes are trivial and the density of metastables is too low to make a significant contribution to the excitation of the excited species under these conditions[9-11]. In a corona model for these levels, we have the electron-impact excitation from the ground-state atom,

$$
\operatorname{Ar}(g s)+e \rightarrow \operatorname{Ar}\left(2 p_{1}\right)+e
$$

and the spontaneous radiation,

$$
\operatorname{Ar}\left(2 \mathrm{p}_{1}\right) \rightarrow \operatorname{Ar}\left(1 \mathrm{~s}_{2}\right)+\mathrm{h} v
$$

The symbol gs denotes the ground state, $1 \mathrm{~s}$ and $2 \mathrm{p}$ denote the Paschen $1 \mathrm{~s}$ and $2 \mathrm{p}$ levels, e is for an electron and $h v$ is for a photon.

The emission intensity, $I$, from the excited state $2 p_{1}$ is given by

$$
\mathrm{I}=\mathrm{A}_{\mathrm{Ar}(2 \mathrm{p} 1)} \cdot n_{\mathrm{Ar}(2 \mathrm{p} 1)}=n_{\mathrm{e}} \cdot n_{\mathrm{gs}} \cdot Q_{\mathrm{exc}}
$$

The symbol $\mathrm{A}$ is the Einstein coefficient, $n$ is the density and $Q_{\text {exc }}$ denotes the excitation rate coefficient[11].

Usually, strong emission lines from the Paschen $2 \mathrm{p}_{1}$ can be observed easily with wavelength of $750.4 \mathrm{~nm}$. Therefore, we choose the intensity of this wavelength to observe its variation with the various process parameters, such as pressure, flow, power and current ratio. The current ratio is ratio of the current of inside coil to the sum current of the both inside and outside coil. Under a low pressure ( $<100 \mathrm{~m}$ Torr), the broadening of the wavelength profile is mainly the instrument broadening as the Doppler broadening and Stark broadening is too low to be observed. We get the intensity of each wavelength by fitting the data from the eight MCSs corresponding to the eight PMTs. The intensity variation under different process conditions is shown in Figure 3 from (a) to (d).

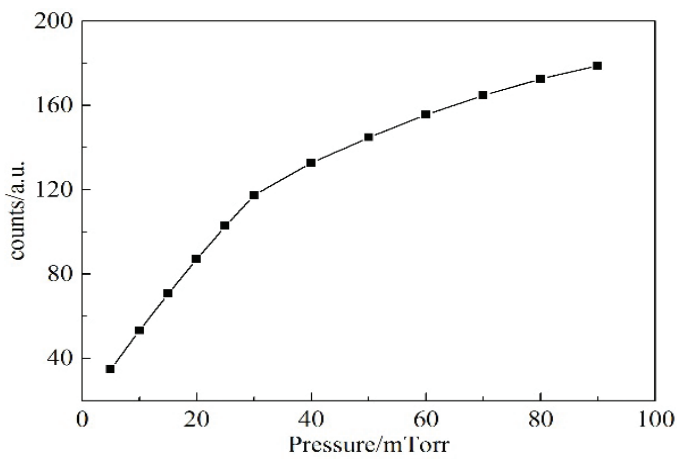

(a) 


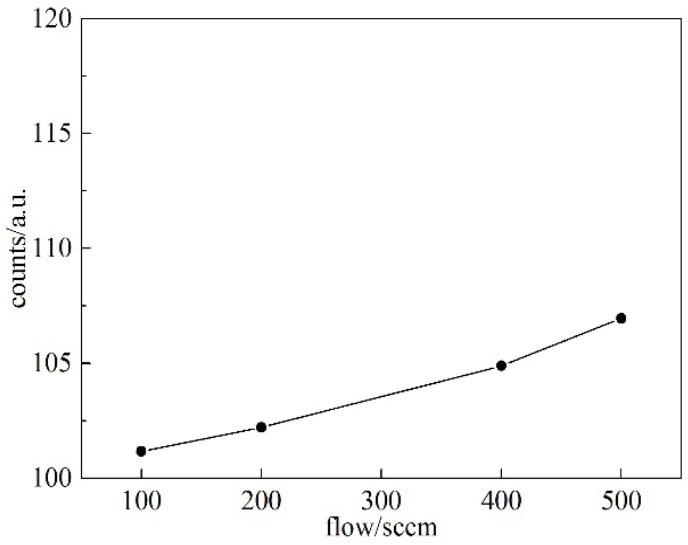

(b)

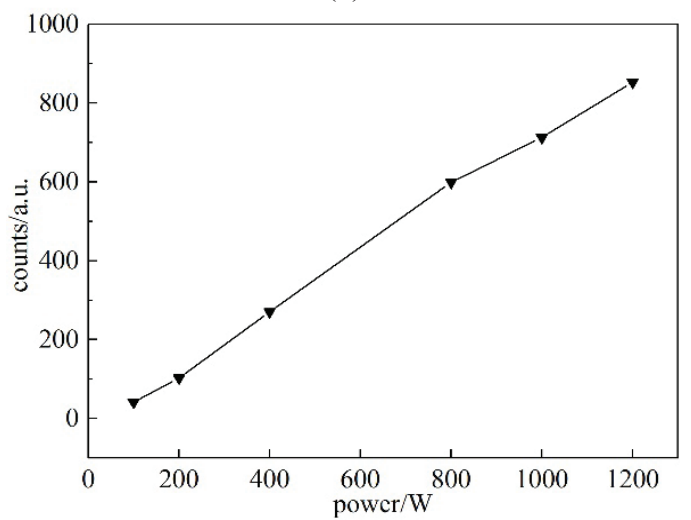

(c)

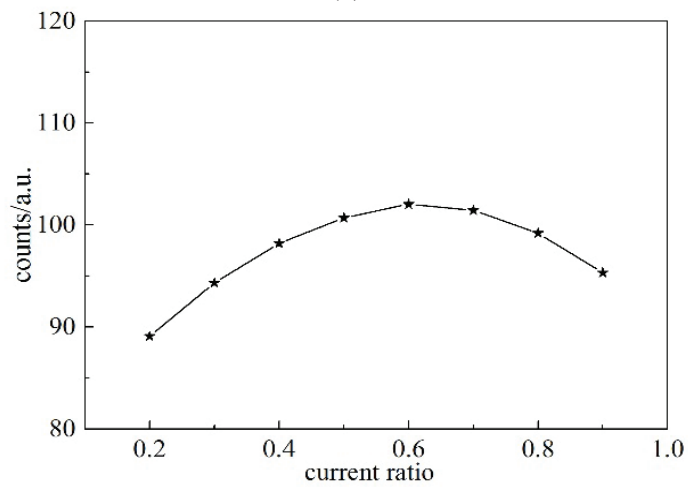

(d)

Fig.3. Intensity of various process conditions

It can be seen from figure 3 that with the increase of processing parameters, the intensity also increases except with the current ratio. As the current ratio increases, the intensity increases firstly and then decreases. And the intensity reaches to maximum value when the current ratio equals to 0.6. Although the intensity almost increases with the process parameters, it barely increases with the gas flow and evidently increases with the pressure and power, especially with power. The reasion lies in the fact that the pressure mainly affects the electronic temperature and power mainly affects electronic density. So while the pressure and power increase, the intensity is also increased due to the increase of electronic temperature and electronic density. With the increase of gas flow, the intensity will increase with the density of Argon gas increases while the pressure is stable. Besides, the variation of coil current ratio merely changes the distribution of plasma in space, which affect the light flux flow into the measurement system. When the current ratio is among $0.5 \sim 0.7$, the plasma distribution in the chamber is uniform, and the measured value of light intensity is higher as a consequence.

\subsection{ICP pulse discharge}

During the pulse discharge, there exists a nonlinear function relation between the any process function $\mathrm{R}$ and discharge power $\mathrm{P}$, as is shown below.

$$
\langle\mathrm{R}[\mathrm{P}(t)]\rangle \neq \mathrm{R}(\langle\mathrm{P}(t)\rangle) \quad\left(\langle X\rangle=\frac{1}{\tau} \int_{0}^{\tau} X \mathrm{~d} t\right)
$$

The symbol $\tau$ is pulse period, $X$ is a arbitrary function, $\mathrm{P}(t)$ is loaded pulse power and angle brackets show the time average value. The significance of equation (4) is that the average value of process function $\mathrm{R}$ does not equal to the value of process function under the time average power. As the equation (4) is nonlinear, the time average processing means inadequacy to depict the key parameters of pulse plasma, such as electronic temperature and density. Therefore, in order to research the pulse discharge plasma, the evolution of the plasma parameters should contain the time dimension. Due to the difficulty to obtain the change of plasma parameters with time, CCD or iCCD is not a suitable tool. However, the PMT has a good time sensitivity to get the light intensity of plasma, and can be used to analyze the light intensity variation with the process parameters. We get the results of the light intensity changes with three different pulse frequencies, as is shown in figure 4 from (a) to (c). And we also obtain the light intensity changes of No.1-8 PMT with time, as is shown in figure 5.

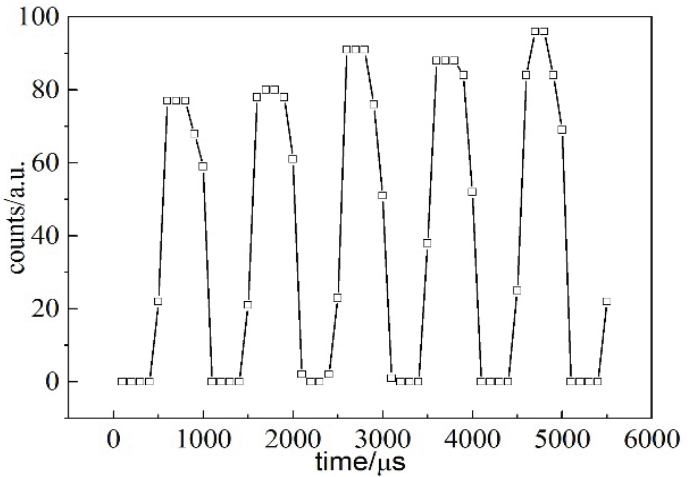

(a)

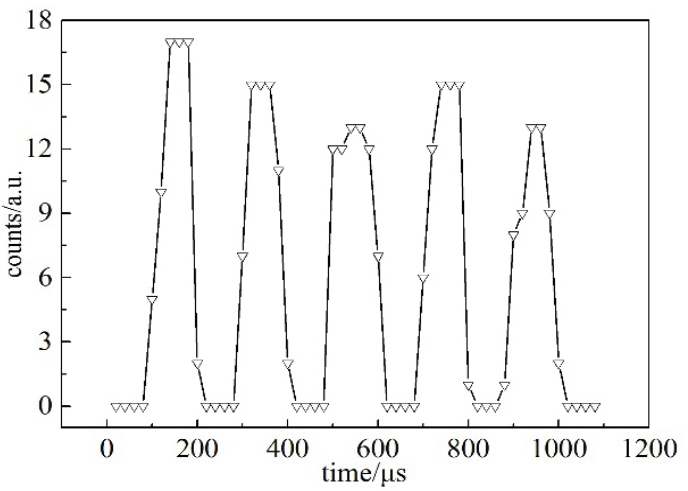

(b) 


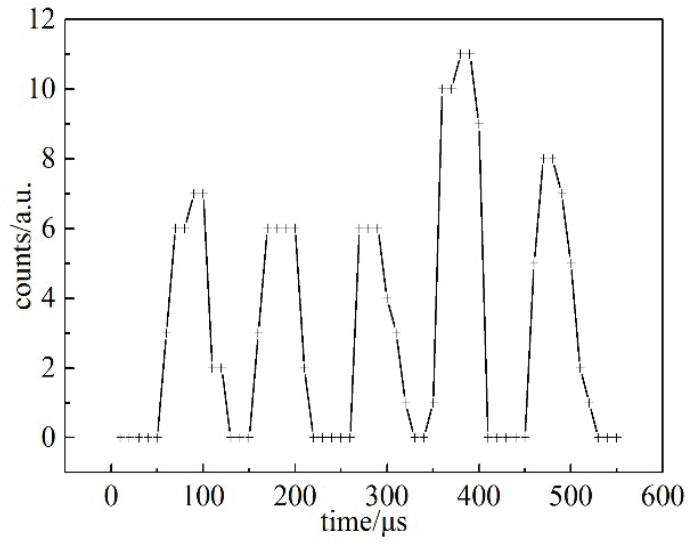

(c)

Fig.4. Intensity change of various pulse frequencies

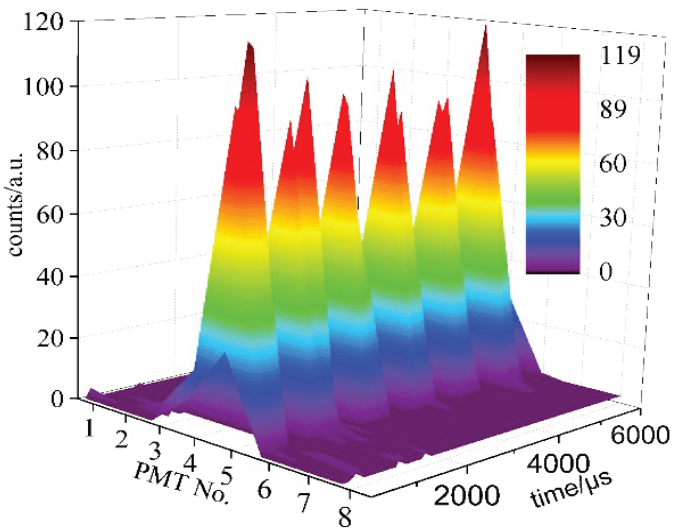

Fig.5. Intensity change of No.1-8 PMT with time increase

Figure 4(a) to 4(c) show that the light intensity varies with the pulse cycle. But the light intensity per 1 millisecond is nearly unchanged when the pulse frequency changes. This is because the change of pulse frequency only changes the number of discharge, which does not affect the light intensity. Increasing the duty ratio of pulse means that the pulse on time is increased, which leads to the improvement of the total light intensity of a pulse cycle. However, when the pulse frequency is constant, changing the duty ratio does not alter the intensity per unit time. Figure 5 shows the intensity change of No.1-8 PMT with a time range of $0 \mathrm{~s}$ to $6 \mathrm{~ms}$. Because of the instrument broadening, the light intensity profile is a Gaussian function distributing among the focal plane of spectrometer and is obtained by the eight vertical array fibers. Figure 5 shows that when the pulse discharge power is loaded by phase step, the light intensity does not change by phase step. Since the light intensity has a relation with plasma parameters, the results in figure 5 show that the plasma parameters evolution is nonlinear during the transient discharge process. Non-stable pulse power supply in pulse discharge steady state leads to unstable collecting spectral intensity, which will also affect the plasma parameters in steady state.

\section{Conclusion}

A measuring system consisted of photo multiplier tubes (PMTs) array, spectrometer and other parts has been developed. And the position of inlet plane of bundle fiber and the focal plane of the spectrometer have been analyzed under two different coordinates and are adjusted to coincide with each other. The output synchronization calibration of the eight PMTs also has been completed. After that, the Ar emission spectrum intensity from typical rf and pulse discharges are collected by this system. The measurement results show that the intensity of Ar emission spectrum increases with the power and pressure but increase less with the gas flow and current ratio. The intensity of the pulsed discharge is almost unchanged with the frequency. Since the light intensity of the pulsed discharge has a relation with plasma parameters, the results show that the plasma parameters evolution is nonlinear during the transient discharge process. For the spectrum broadening in low pressure is too narrow to be observed. The broadening is mainly the instrument broadening. However, the spectrum broadening in high pressure discharge is the convolution of Doppler broadening and Stark broadening and is wide enough to be observed, so we can make use of this system to obtain the spectrum profile so as to analyze the ion temperature, which will be our further work.

The authors thank Profess Pu Yikang of Tsinghua University for his help in the experiment and data analysis. This work is financially supported by National Science and Technology Major Project (2011ZX02403-4$3)$.

\section{References}

1. E. Karakas, V. M. Donnelly, and D. J. Economou, J. Appl. Phys. 21, 870-876 (2013)

2. M. B. Chowdhuri, J. Ghosh, R. Manchanda et al. Rev. Sci. Instrum. 85, 11E411-11E411-3 (2014)

3. S. Kamio, N. Suzuki, Q. H. Cao et al. Rev. Sci. Instrum. 83, 083103 (2012)

4. R. M. Lan, X. F. Liu, X. R. Yao, W.K. Yu, G.J. Zhai, J. Optoelectronics. Laser, 26, 2175-2179 (2015)

5. T. H. Chung, H. R. Kang, M. K. Bae, Phys. Plasmas 19, 113502 (2012)

6. Y. Chen, Z.G. Guo and X.M. Zhu, J. Phys. D: Appl. Phys. 40, 5112-5116 (2007)

7. G. Avaria, O. Cuadrado, J. Moreno, C. Pavez, L. Soto, J. Phys.: Conf. Ser. 720, 012041 (2016)

8. U. Fantz, Plasma Sources Sci. Technol. 15, S137S147(2006)

9. J. B. Boffard, C. C. Lin, C. A. J. Phys. D: Appl. Phys. 37, R143-61 (2004)

10. V. M. Donnelly, J. Phys. D: Appl. Phys. 37, R21736 (2004)

11. J. T. Gudmundsson, E. G. Thorsteinsson, Plasma Sources Sci Technol. 16, 399-412 (2007) 